\title{
Diseño probabilístico de estructuras de madera en Norteamérica
}

\author{
Raymundo Dávalos Sotelo ${ }^{1}$
}

\begin{abstract}
RESUMEN
Se revisa el tema del diseño basado en la confiabilidad en Norte América. Se describe el objetivo del diseño probabilístico usando métodos de primer orden y Segundos Momentos, basados en el cálculo del índice de confiabilidad, a partir de cual se puede estimar la probabilidad de falla $P_{\mathrm{f}}$. Se discuten los procedimientos de calibración de las ecuaciones de diseño. Se presentan los factores de carga especificados por algunos reglamentos para combinaciones de carga usuales y se incluyen los factores de reducción de resistencia correspondientes. Un argumento a favor del diseño probabilístico es que provee una medida cuantitativa de la seguridad. Sin embargo, las diferencias entre las tasas observadas de falla y las predichas a menudo son substanciales. Se comentan algunas de las razones que podrían explicar dichas discrepancias. Se argumenta que los requisitos de servicio de los códigos actuales podrían ser insuficientes para garantizar un comportamiento funcional de las estructuras modernas.
\end{abstract}

\section{PALABRAS CLAVE}

Diseño por estados límite, índice de confiabilidad, probabilidad de falla, factores de carga, factores de resistencia, factor de duración de carga.

\begin{abstract}
The subject of reliability-based design for North America is reviewed. The objective of probabilistic design using First order Second moment methods is described. These Methods are based on the concept of safety index, from which the probability of failure, $P_{f}$, can be estimated. Calibration procedures for the design equations are discussed. Load factors specified by different codes for usual loading combinations are presented, as well as the resistance-reducing factors. One argument in favor of probabilistic design is that it provides a quantitative measurement of the safety of structures. However, quite often, the differences between observed and predicted failure rates are substantial. Some of the reasons for these discrepancies are presented. Results from the literature indicate that the serviceability requirement of the current codes could be insufficient in terms of reliably predicting the functional behavior of modern structures.
\end{abstract}

1 Investigador. Departamento de productos forestales y conservación de bosques. Instituto de Ecología, A.C. Apdo. Postal 63. 91000 Xalapa, Ver. México.

Manuscrito recibido para su publicación el 8 de Febrero de 1994. 


\section{INTRODUCCION}

La madera es un importante material que se usa en construcción en casi todos los países del mundo y la mayoría de los tipos de estructuras. Por ejemplo, en 1987 en los Estados Unidos se utilizaron en la construcción más productos a base de madera (por un valor de 40.4 miles de millones de dólares) que todos los otros materiales importantes combinados (metales, concreto, yeso y tejas cerámicas: 37.5 miles de millones de dólares) (USDC, 1991). La madera es, además, un material de interés internacional. Se comercializa más extensamente a este nivel que cualquier otro material estructural (Bodig, 1992). Entre otras ventajas de la madera, desde el punto de vista de su uso como material de construcción, se sabe que es un recurso natural renovable que requiere poca energía para su conversión en componentes estructurales (Boyd, et al.,1976) y que es un recurso que provee beneficios ecológicos deseables comparado con otros materiales alternativos. Estos atributos aseguran el futuro de la madera como un importante material estructural.

En este trabajo se revisa un tema relacionado con el uso estructural de la madera: el diseño basado en la confiabilidad. Se pretende presentar un estado del arte en Norte América, incluyendo la República Mexicana. Las fuentes principales usadas en la elaboración del presente documento para describir la situación en Estados Unidos y Canadá son las Memorias de un Taller de Investigación Avanzada sobre Diseño de Estructuras de Madera basado en la confiabilidad (TIA-OTAN), el cual fue patrocinado por la organización del tratado del Atlántico del Norte (Bodig, 1992) y, el informe No. 34 del Departamento de Ingeniería Civil de la
Universidad de la Columbia Británica (Foschi, et al., 1989). Para el caso de México, se consultó la información generada en el Departamento de Productos Forestales y Conservación de Bosques del Instituto de Ecología, A.C. (Dávalos, et al., 1977; Echenique, et al., 1987; Robles, et al., 1988) y se revisaron los trabajos de Meli (1976, 1985 y 19991).

\section{CONCEPTOS FUNDAMENTALES}

Los procedimientos basados en la confiabilidad para el diseño de estructuras están siendo desarrollados y logrando la aceptación de los organismos responsables de los reglamentos de construcción en todo el mundo. Estos reglamentos contienen cláusulas para garantizar que la estructura sea segura bajo la acción de cargas extremas y que permanezca funcional bajo las cargas de servicio. En forma de ecuación, esto se expresa de la siguiente manera:

$$
\begin{aligned}
R_{d} & \geq S_{d} \\
\Delta_{\text {perm }} & \geq \Delta_{\text {serv }}
\end{aligned}
$$

Donde:

$\mathrm{R}_{\mathrm{d}} \quad=$ resistencia de diseño

$\mathrm{S}_{\mathrm{d}} \quad=$ efecto de cargas de diseño

$\Delta_{\text {perm }}=$ deflexión aceptable

$\Delta_{\text {serv }}=$ efecto de cargas de servicio

El modelo conceptual de diseño probabilístico está basado en la teoría de confiabilidad clásica (ver por ejemplo, Freudenthal, et al., 1966; Shinozouka, 1983). El objetivo general de diseño basado en la confiabilidad es tomar en cuenta la incertidumbre de las variables involucradas, utilizando los principios de la teoría de probabilidad, y dimensionar la estructura para permitir un riesgo tolerable de fallas en su comportamiento (probabilidad de falla) (Foschi, et al., 1989). 


\section{EVOLUCION DE LOS CRITERIOS DE VERIFICACION DE LA SEGURIDAD}

Históricamente, los reglamentos de diseño se han basado en la experiencia profesional, el juicio y la intuición de los calculistas. En los últimos 40 años, se han logrado una serie de avances considerables, por ejemplo: la adopción del los métodos de Diseño por Estados Límite y, la introducción de la computadora como una herramienta rutinaria de análisis y diseño entre los más importantes. Sin embargo, a pesar de los avances recientes en computación aplicada a la mecánica, las complejas interrelaciones entre cargas y resistencias son todavía difíciles de predecir con exactitud (Ellingwood, 1992). Las incertidumbres más importantes están relacionadas con la variabilidad en las cargas, la variabilidad en la resistencia, la variabilidad en las dimensiones de los elementos, la súbita aparición de riesgos naturales (sismos, huracanes, etc.), los riesgos por actividades humanas que son enormemente difíciles de predecir, amén del conocimiento insuficiente acerca del comportamiento de los materiales y los inevitables errores humanos en el diseño y la construcción.

Con el advenimiento de los primeros métodos de diseño desarrollados en el siglo XIX, se introdujo el concepto de factor de seguridad. Estos métodos estuvieron basados en conceptos elásticos y tomaron la forma de lo que ahora se conocen como métodos de diseño por esfuerzos permisibles. La idea básica de estos métodos fue (y todavía es) seleccionar las cargas de manera conservadora, calcular los esfuerzos elásticos, y verificar que estos esfuerzos fueran menores que una fracción considerada segura de los esfuerzos resistentes (Ellingwood, 1992). En forma de ecuación:

$$
f_{k}=\frac{F_{k}}{F S}
$$

en la cual $f_{k}$ es el esfuerzo debido a cargas aplicadas, $F_{k}$ es el esfuerzo resistente y FS es el factor de seguridad. El factor de seguridad representa el nivel de confianza que se tiene en los métodos de diseño de vigor, así como en el conocimiento del comportamiento de los materiales. Los valores de los factores de seguridad han ido disminuyendo más o menos en forma continua desde finales del siglo XIX. Por ejemplo, en el caso del acero, en 1880 el valor de FS era de 2.5 y en la década de 1940, había disminuido a 1.67, donde ha permanecido hasta la fecha (Ellingwood, 1992).

En las décadas de 1940 y 1950 surge el concepto de regularidad estadística de las variables de carga y resistencia y se plantea por primera vez que la incertidumbre puede ser descrita cuantitativamente por distribuciones de probabilidad. El Prof. Freudenthal sugirió que la probabilidad de falla $P_{f}$ debería usarse en vez de FS como una medida cuantitativa de la seguridad y respuesta estructural de las construcciones (Freudenthal, et al., 1966).

En otros avances, el Comité 318 del American Concrete Institute introdujo en 1963 el diseño por resistencia última como alternativa al método de esfuerzos permisibles. Los métodos de confiabilidad formales todavía no se introdujeron en esa época. En 1969 las organizaciones involucradas en los Estados Unidos en el desarrollo de métodos de diseño para estructuras de acero, iniciaron los trabajos para desarrollar los métodos probabilísticos. En aquel país estos métodos se conocen como Métodos de "Diseño por Factores 
de Carga y Resistencia" (DFCR). Las especificaciones resultantes de estos trabajos no se publicaron sino hasta 1986, ¡17 años después! de que los trabajos se iniciaron, lo que da una idea de la dificultad que se encontró en lograr un formato aceptable para la profesión (AISC, 1986). En 1979 se iniciaron los trabajos para desarrollar factores de carga uniformes para todos los materiales. Estos trabajos culminaron con la publicación de la norma American National Standard ANS A58.1-1982 ("Cargas de Diseño Mínimas para Edificios y Otras Estructuras"), actualmente conocida como Norma ASCE 7-88 (ASCE, 1990).

\section{ASPECTOS DE DISEÑO ESTRUCTURAL CON MADERA}

Entre los factores que consideran los ingenieros para la selección de los materiales estructurales están las consideraciones de tipo económico, la disponibilidad del material y la confianza del diseño. En los países con un alto grado de desarrollo en la ingeniería estructural de la madera, ésta recibe una calificación bastante alta en los dos primeros rubros. Sin embargo, aún en estos países, no sucede así en el tercer punto mencionado, especialmente ahora que el acero y el concreto tienen reglamentos basados en métodos de diseño por estados límite en la mayoría de los países del mundo. La madera presenta una serie de problemas como un material estructural que no enfrentan ni el concreto ni el acero. Tal vez el principal de ellos sea la gran variabilidad inherente del material, la cual se logra reducir, únicamente en parte, con la clasificación estructural. Por otro lado, la construcción con madera incluye una amplia gama de productos (laminados, contrachapados, aglomerados, etcétera) con propiedades estructurales bastante diferentes. Además, muchas de las ecuaciones que se emplean en el diseño de miembros y conexiones son obsoletas. Por si lo anterior fuera poco, la mayoría de las escuelas no enseñan diseño estructural con madera (Ellingwood, 1992).

Otro aspecto particular de la madera lo constituye el efecto de duración de carga (DC) sobre la resistencia. Desde hace varias décadas se sabe que la madera es un material sensitivo a la tasa de aplicación de la carga así como a la duración misma de la carga, asociándose una mayor resistencia a tasas más rápidas y duraciones más cortas (Wood, 1951).Tradicionalmente, en muchos reglamentos de diseño del mundo, se han utilizado como base los resultados de los experimentos de Wood (1951) para cuantificar el efecto de la duración de la carga sobre la resistencia. Estos experimentos se hicieron con pequeños elementos a flexión libres de defectos. En la década de los 70's, se empezó a acumular evidencia que este efecto era diferente para las pequeñas probetas y para la madera comercial con defectos (Madsen y Barrett, 1976).Desde entonces, varios programas de investigación han desarrollado enfoques diferentes para tratar este problema (Barrett y Foschi, 1979; Nielsen, 1984, Gerhards y Link, 1986).

\section{ANALISIS DE CONFIABILIDAD}

Una situación dada de diseño siempre puede describirse matemáticamente en términos de una función $G(\mathbf{X})<0$ que relacione las variables de carga $y$ resistencia para el estado límite específico de interés. Este modelo,

$$
G\left(X_{1}, X_{2}, \ldots, X m\right)=0
$$

en el cual $\mathbf{X}=\left(\mathrm{X}_{1}, \mathrm{X}_{2}, \ldots, \mathrm{X}_{\mathrm{m}}\right)$ representa las variables de carga y resistencia, está 
definido por convención de tal forma que la falla ocurre cuando $\mathrm{G}(\mathbf{X})<0$. En el contexto de confiabilidad estructural, los estados límite se clasifican comúnmente ya sea como estados límite de resistencia o últimos 0 , como estados límite de servicio. Los primeros corresponden a los límites de capacidad de carga de toda o parte de una estructura, por ejemplo, exceder la resistencia del material de un elemento, fractura, fatiga, inestabilidad o volteo. Los estados límite de servicio corresponden a la perturbación del uso funcional de una estructura, por ejemplo, grietas excesivas, deformaciones y vibraciones (Foschi, et al., 1989).

La probabilidad de alcanzar un estado límite dado 0 , probabilidad de falla, $\mathrm{P}_{\mathrm{f}}$, se obtienen calculando la probabilidad del evento $G<0$. Puesto que, en general, se encuentran involucradas muchas variables en la función $G$, este cálculo requiere la función de densidad de probabilidad conjunta y una integración múltiple sobre la región de falla $\mathrm{G}<0$ (Foschi, et al., 1989). Matemáticamente,

$$
P_{f}=\int f_{x}\left(x_{1}, x_{2}, \ldots, x_{m}\right) d x_{1} d x_{2} \ldots d x_{m}
$$

en el cual $f_{x}(\mathbf{x})$ es la función de densidad conjunta de $\mathbf{X}$ y el dominio $\mathrm{D}$ es la región de $\mathbf{x}$ donde $\mathbf{G}(\mathbf{X})<0$ (Ellingwood, 1992).

La ecuación (5) es difícil de calcular para estructuras reales, dado que no se conoce de antemano la función de densidad de probabilidad requerida, además de que es muy difícil de obtener por medios exactos. En repuesta a estos problemas se desarrollaron los Métodos de Primer Orden y Segundos Momentos (Madsen, et al., 1986). Estos métodos se basan en el cálculo de un índice de confiabilidad, â, a partir del cual se puede estimar la probabilidad de falla $P_{f}$ utilizando la función de distribución de probabilidad normal estándar. El índice de confiabilidad, â, está definido por,

$$
\beta=\frac{m_{G}}{\sigma_{G}}
$$

en el cual, $m_{G \_}$y $o_{G}$ son la media y la desviación estándar de la función estado límite, $\mathbf{G}(\mathbf{X})$, linearizada en un punto de expansión apropiado de la superficie de falla $G(\mathbf{X})=0$. Esta definición de â es válida solamente cuando $G$ es una variable normal. Para esto se necesita que $G$ sea una combinación lineal de variables normales no correlacionadas.

Se puede demostrar que el índice de confiabilidad, â, es la distancia mínima entre el origen, $\mathbf{x}=\mathbf{0}$ (correspondiente al valor promedio de $\mathbf{X}$ ) y la superficie de estado límite $G(\mathbf{X})=0$ como se ilustra en la figura 1 para el caso de dos variables $\mathrm{X}_{1}$, y $\mathrm{X}_{2}$ (Foschi, et al., 1989). Esta interpretación geométrica permite el desarrollo de algoritmos de computadora iterativos para el cálculo (Madsen, et al., 1986). El punto $\mathbf{x}^{\star}$ sobre la superficie de estado límite que está más cercano al origen se llama punto de diseño (Foschi, et al., 1989). El índice de confiabilidad, â, y la probabilidad de estado límite de falla, $\mathrm{P}_{\mathrm{f}}$, están relacionados, en forma aproximada, como se indica en las ecuaciones (7) y (8):

$$
\begin{gathered}
P_{f}=\phi(-\beta) \\
\beta=\phi^{-1}\left(1-P_{f}\right)
\end{gathered}
$$

en las cuales $\Phi(\bullet)$ es la integral de probabilidad normal estándar (Ellingwood, 1992). La definición más precisa de â es 


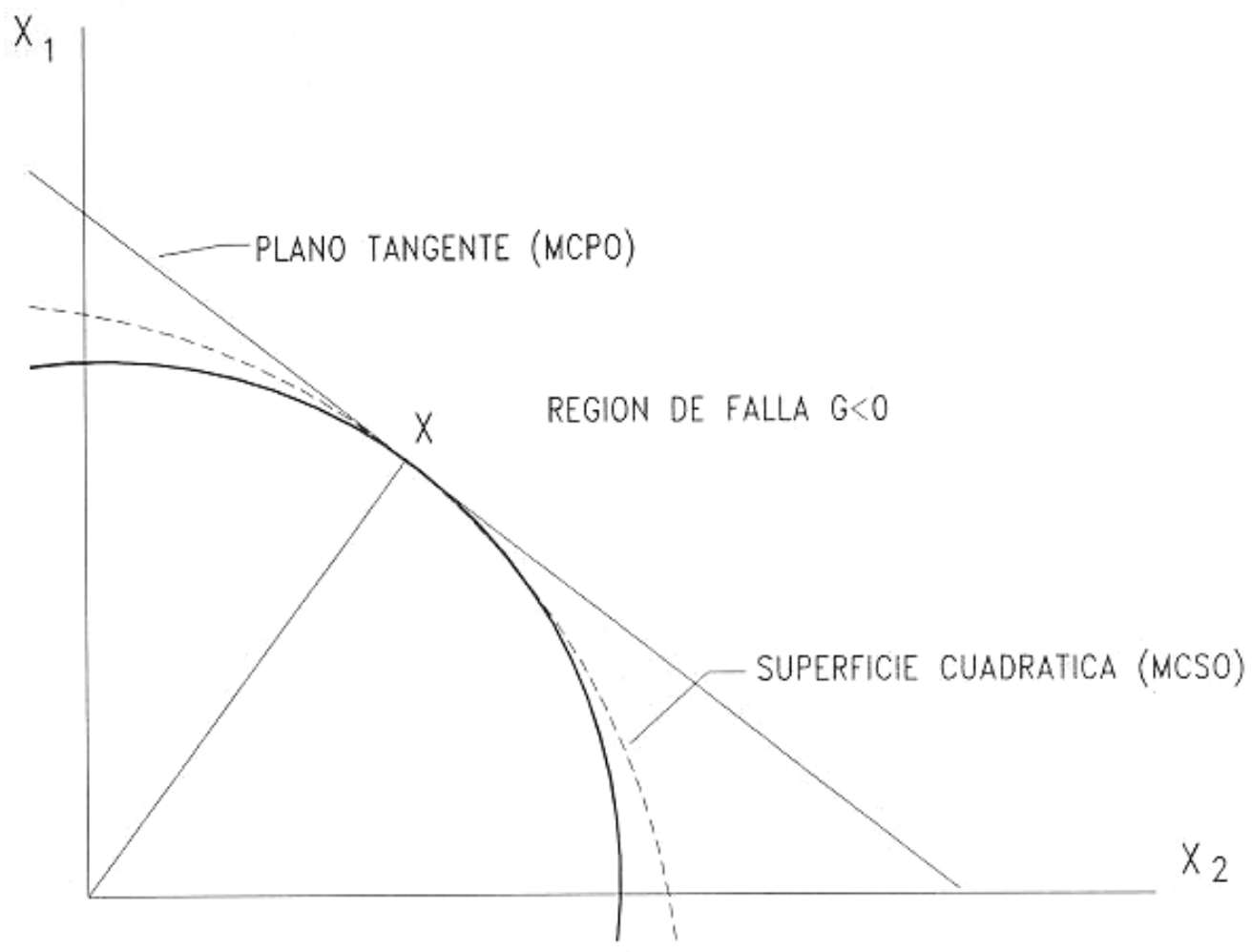

Figura 1. Representación geométrica de los cálculos de confiabilidad (Foschi, et al., 1989)

la dada por la ecuación (7). Para obtener esta $\hat{a}$ hay que normalizar todas las variables, hacerlas no-correlacionadas y luego, encontrar la distancia mínima entre el origen (valor medio de las variables iniciales) y la superficie $G(\mathbf{X})=0$. Si esta superficie fuera lineal, entonces la ecuación (7) sería exacta. La estimación de $P_{f}$ es de hecho exacta si todas las variables que intervienen están distribuidas normalmente y si se combinan linealmente en la función G. Generalmente las variables no son normales y la función $G$ no es lineal (Foschi, et al., 1989). En este caso, se trata de una aproximación, que es el caso más común.

\section{Resistencia de la madera}

La aplicación de procedimientos basados en la confiabilidad depende de un conocimiento detallado del comportamiento del material. Para análisis de la confiabilidad es preciso representar con suficiente precisión la cola inferior de la distribución. En la figura 2, reproducida de Foschi, et al., (1989), se presentan datos de resistencia de la madera a los que se les ajustó una distribución Weibull de dos parámetros en dos formas diferentes: con la línea interrumpida se presenta el ajuste obtenido con el $100 \%$ de los datos, mientras que con línea sólida se indican los resultados del ajuste al $15 \%$ 
inferior de la distribución. Esta figura muestra que un ajuste Weibull de dos parámetros utilizando el $100 \%$ de los datos no representa de manera exacta la distribución de los mismos debajo del nivel del $10^{\circ}$ percentil.

Por el contrario, la misma distribución Weibull de dos parámetros ajustada a los datos truncados al $15^{\circ}$ percentil muestra un muy buen ajuste para esta porción de la distribución lineal (Foschi, et al., 1989). Tomando en cuenta los resultados mostrados en la figura 2, resulta evidente que los métodos de prueba de carga son satisfactorios para representar la resistencia de la madera estructural (Foschi, 1992).

\section{Modelos de la duración de la carga}

Como se ha indicado, la resistencia de la madera depende del tiempo de aplicación de la carga y la falla puede ocurrir por deformación excesiva o por sobrecarga. Así que debe conocerse la historia completa de carga para efectuar un análisis de confiabilidad (Ellingwood, 1992). Puesto que los experimentos de laboratorio se conducen con cargas incrementadas linealmente o bajo cargas constantes, se requieren formas de extrapolar los resultados para cargas de servicio intermitentes, que se puedan aplicar en análisis de confiabilidad. Esto se ha logrado utilizando modelos de acumulación de daño para crecimiento lento de grietas. Un modelo desarrollado en los Estados Unidos describe la tasa de daño como una función exponencial (Gerhards y Link, 1986). Los modelos desarrollados en Canadá son función de los daños existentes así como del esfuerzo actuante (Barret y Foschi, 1979; Foschi et al., 1989). Los tiempos de falla bajo esfuerzo constante predichos por los cuatro diferentes modelos, se ilustran en la figura 3. Todos los experimentos citados se han conducido utilizando cargas constantes, lo cual limita la aplicabilidad de los mismos al análisis de combinaciones de carga de tipo estática. La aplicación de cualquiera de estos modelos a las combinaciones de carga que involucren viento o sismo es cuestionable (Ellingwood,1992).

Un modelo diferente ha sido propuesto por Nielsen (1984), considerando el lento crecimiento de grietas en un material con propiedades viscoelásticas alrededor del extremo de la grieta. Este modelo utiliza una función de flujo de fluidos, que intentan relacionar el flujo de los esfuerzos con el crecimiento de las grietas y el daño. Puesto que la velocidad de crecimiento de la grieta es proporcional a la longitud presente de la grieta, el modelo de Nielsen también muestra un crecimiento exponencial similar al de los otros modelos.

\section{DISEÑO POR ESTADOS LIMITE}

Un componente o sistema estructural puede ser definido por sus propiedades de resistencia característica,sus dimensiones y cargas, expresadas en forma de vector como $\mathbf{x}_{\mathbf{n}}$. La probabilidad de alcanzar un estado límite es una función de estas variables y, de esta manera la respuesta estructural planteada por los reglamentos se expresa en términos probabilísticos como se indica en la ecuación (9) (Ellingwood,1992):

$$
\beta\left(x_{n}\right)=\phi^{-1}\left[1-P_{f}\left(x_{n}\right)\right]>\beta_{0}
$$

en la cual $\hat{a}_{0}$ es el índice de confiabilidad buscado. El objetivo del diseño probabilístico es llegar a un diseño aceptable, expresado por el conjunto de variables de diseño, $\mathbf{x}_{\mathbf{n}}$, utilizando ecuaciones de diseño convencionales con 


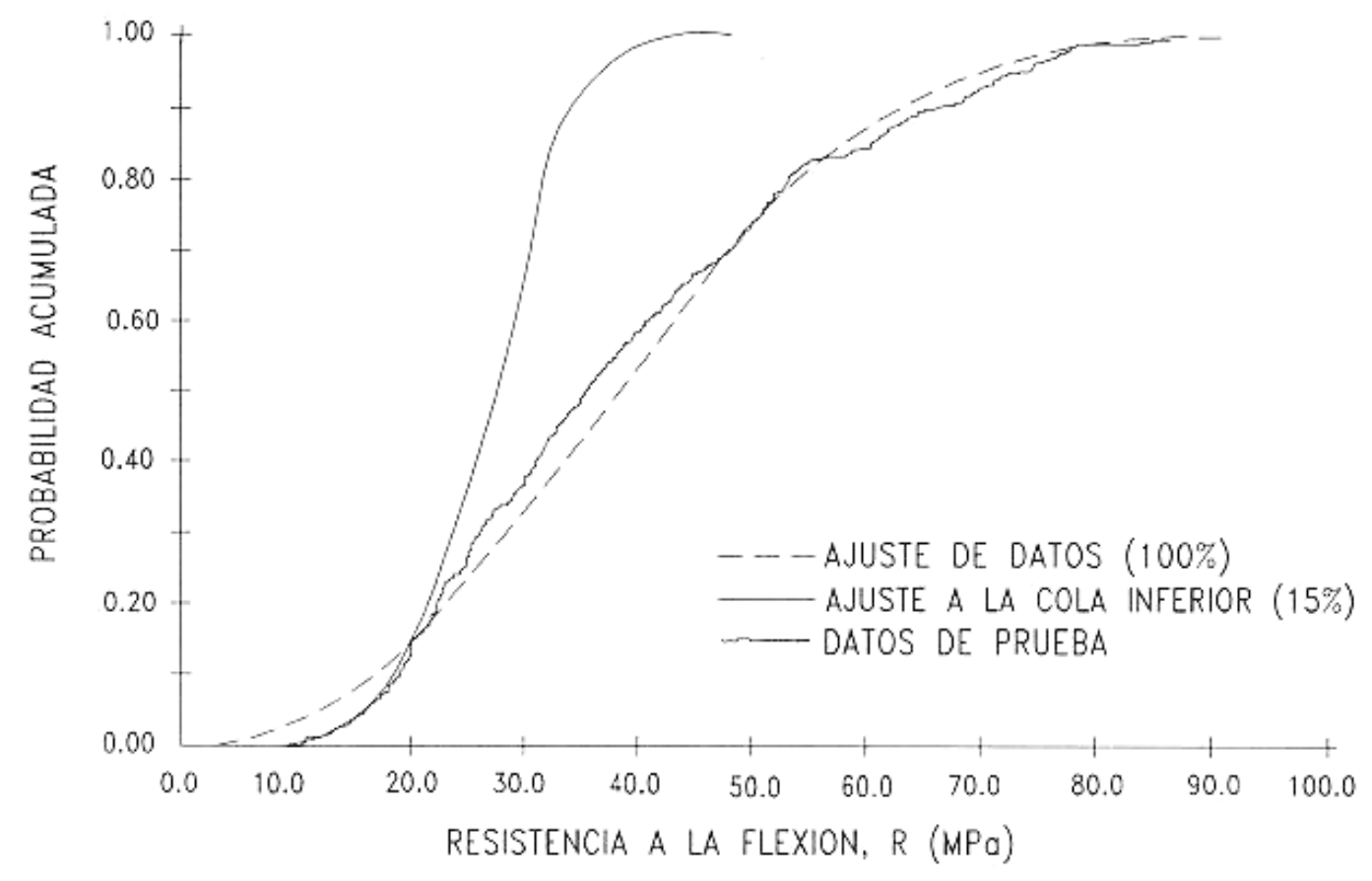

Figura 2. Dos tipos de ajuste con distribución Weibull de dos parámetros ( $100 \%$ de los datos vs $15 \%$ de Iso mismos) (Foschi et al., 1989)

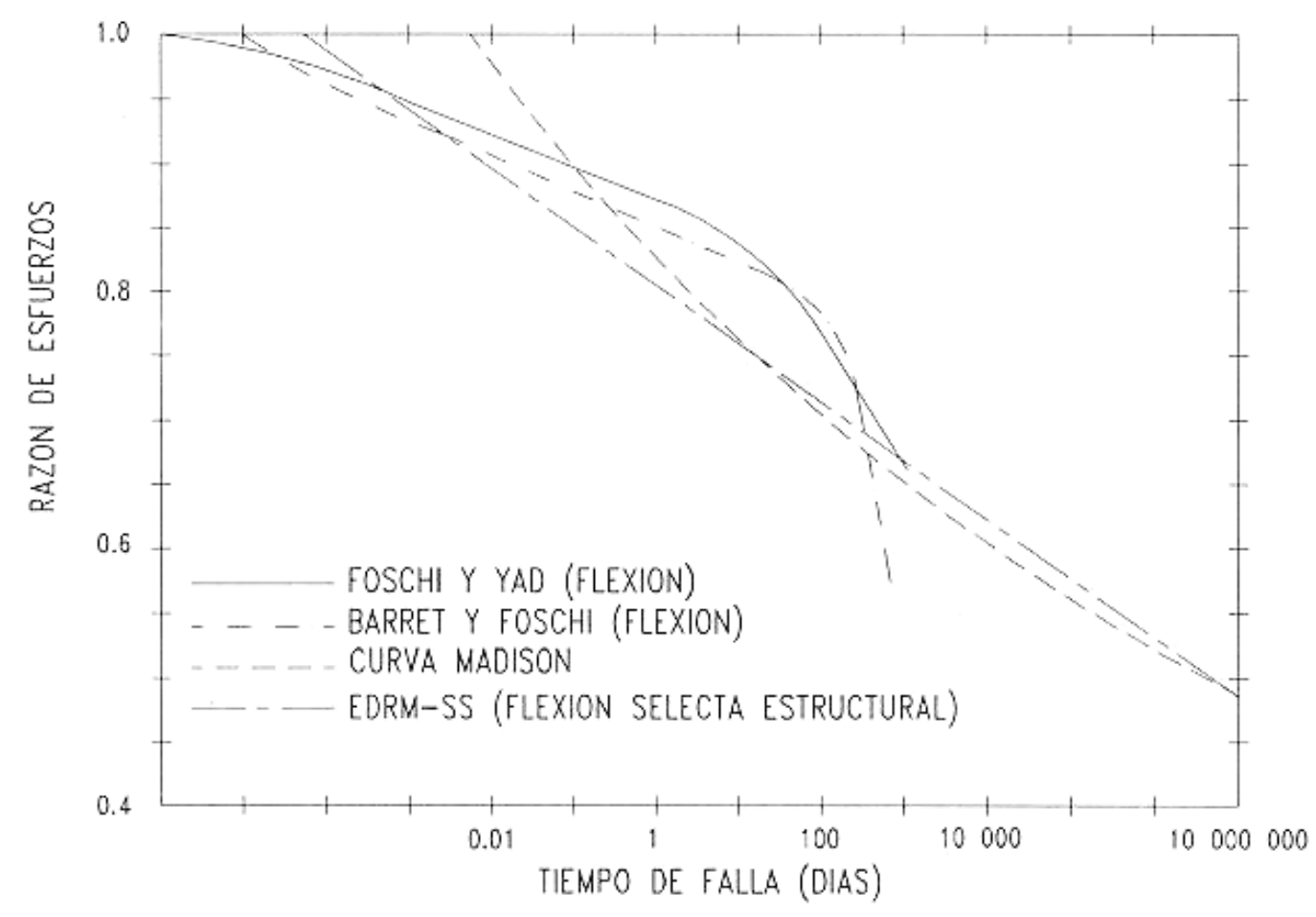

Figura 3. Comparación de cuatro modelos para predecir el tiempo de falla de los Elementos de madera bajo esfuerzos constantes (Ellingwood, 1992) 
la forma de la ecuación (10) (Ellingwood, 1992):

$$
K_{D} F_{R} R_{n}>\sum F_{C i} Q_{n i}
$$

en la cual

$$
\begin{aligned}
& \mathrm{K}_{\mathrm{D}}=\text { factor de efecto de tiempo } \\
& \mathrm{F}_{\mathrm{R}}=\text { factor de resistencia } \mathrm{o} \\
& \text { comportamiento } \\
& \mathrm{R}_{\mathrm{n}}=\text { resistencia nominal } \\
& \mathrm{F}_{\mathrm{ci}}= \text { factor de carga } \\
& \mathrm{Q}_{\mathrm{ni}}=\text { carga nominal }
\end{aligned}
$$

La transformación entre las ecuaciones (9) y (10) es efectuada por un comité encargado de la elaboración del reglamento; a este proceso se le llama "calibración". La calibración de las ecuaciones de diseño basadas en la confiabilidad requiere de la definición de índices de confiabilidad para diferentes aplicaciones. Por ejemplo, en las dos versiones más recientes del Reglamento de Construcciones para el Distrito Federal (DDF, 1976; DDF, 1987a) se emplea la definición de Rosenblueth y Esteva (1973) para el índice de confiabilidad $\hat{a}$; en este planteamiento, la variable, $\boldsymbol{v}$, que relaciona la resistencia, $R$, y las cargas, $Q$, y mide la seguridad de la estructura es logarítmiconormal (ecuación (11)):

$$
v=\ln (R / Q) \text {, si } \quad v<0 \quad \text { hay falla }
$$

Aplicando las aproximaciones de Primer Orden y Segundos Momentos, el índice de confiabilidad obtenido se expresa como señala la ecuación (12):

$$
\beta=\frac{\ln \left(\frac{m_{R}}{m_{Q}}\right)}{\sqrt{C_{R}^{2}+C_{Q}^{2}}}
$$

donde

$$
\begin{aligned}
m_{R}= & \text { valor medio de la resistencia } \\
m_{Q}= & \text { valor medio del efecto de las } \\
& \text { cargas actuantes } \\
C_{R}= & \begin{array}{l}
\text { coeficiente de variación de la } \\
\text { resistencia }
\end{array} \\
C_{Q}= & \begin{array}{l}
\text { coeficiente de variación del efecto } \\
\text { de las cargas actuantes }
\end{array}
\end{aligned}
$$

La ecuación (12) proporciona el valor correcto de â solamente si las variables $R$ y $Q$ son lognormales. Más aún, la ecuación 12 es una aproximación útil cuando los coeficientes de variación de $R$ y $Q$ no son muy grandes (menores a $0.30)$. En casos en que las variables $R$ y $Q$ estén descritas por otras distribuciones (por ejemplo Weibull), la ecuación (12) no daría entonces el valor correcto de â, el cuál tendría que ser calculado con métodos más sofisticados (los métodos de confiabilidad descritos por Madsen et al., 1986).

\section{Indice de confiabilidad buscado}

En el desarrollo de un reglamento para estructuras de madera basado en la confiabilidad, deben tenerse en mente dos objetivos: a) lograr compatibilidad con niveles de confiabilidad de otros materiales para la misma aplicación estructural, y b) compatibilidad con la experiencia previa (Foschi, et al.,1989). En los reglamentos mexicanos se han tomado los valores del índice de confiabilidad, â = 3.9 para estructuras típicas y estados límite de falla dúctil $y, \hat{a}=4.5$ para estructuras cuya falla tenga consecuencias excepcionalmente graves y para estados límite de falla frágil, con base en la deducción de los índices de confiabilidad implícitos en reglamentos anteriores y de conformidad con otros reglamentos internacionales (Meli, 1976). Estos valores de â se calculan en la ecuación (12). 
Meli 1976 llama la atención sobre el hecho de que se podría haber obtenido una confiabilidad más uniforme si se hubiera empleado factores de carga distintos para cargas permanente (muerta) y para cada variable (viva), ya que para razones, $r$, de carga muerta a carga total (muerta más viva) muy bajas $(r<0.5)$, el diseño con los factores de carga del reglamento del $D F$ de lugar a una confiabilidad significativamente menor a la deseada (Nota; las cursivas son del autor). El argumento planteado por Meli (1976) es la confiabilidad para cargas vivas relativamente altas, $r<0.5$, es significativamente menor que cuando domina la carga muerta por la mayor incertidumbre de la carga viva. La forma de hacer más uniforme la confiabilidad sería empleado factores de carga mayores para cargas variables que para cargas permanentes. Sin embargo, para el caso del Reglamento del DF (DDF, 1976; DDF, 1987a) se consideró que la mayor sencillez que se logra en el diseño justificaba la adopción de un factor de carga constante (Meli, 1976).

En el caso de las estructuras de madera, en las Normas Técnicas Complementarias para Estructuras de Madera (DDF, 1987b), los valores de â adoptados, siguiendo el enfoque de Meli (1976) sonde 3.9 para flexión (especies de coníferas, latifolidas y madera contrachapada de pino) y de 4.5 para tensión y compresión (especies coníferas). La fórmula de cálculo es la misma que se aplicó para los otros materiales (Ec. 12), aunque la variabilidad de la madera es mayor. Los valores de los coeficientes de variación para especies mexicanas se tomaron de Echenique-Manrique, et al. (1987) para las especies coníferas $\left(C_{R}\right.$ varía de 0.18 a 0.26 , para madera clasificada según las normas mexicanas), de Cerón (1988) para latifoliadas $\left(C_{R}\right.$ promedio de 0.19) y de Chávez (1985) para madera contrachapada $\left(C_{R}=0.17\right)$. De acuerdo con Foschi (1979), los errores introducidos al emplear la ecuación 12 en casos en que el coeficiente de variabilidad es menor a 0.30, no exceden del $10 \%$, lo cual parece aceptable.

Los diseños resultantes permiten construir estructuras con niveles de confiabilidad similares a las construidas anteriormente tanto en México como en otros países (Dávalos, et al., 1977), con lo que se cumple el requisito de compatibilidad con la práctica previa y, también se logran diseños comparables a los que actualmente se generan en los otros países norteamericanos (Dávalos 1994).

Como comparación, el valor promedio de â utilizado en el Reglamento de construcciones canadiense (NRC, 1985) es de 2.6 para estructuras de madera y de acero (Foschi, et al., 1989), bastante menor al empleado en los reglamentos mexicanos. Las diferencias deben venir del modo de cálculo del índice â. Los reglamentos mexicanos aún emplean como base de sus cálculos, la definición de â de Rosenblueth y Esteva (1973) mientras que otros reglamentos en Norte América (por ejemplo: NRC, 1985; AISC, 1986; ACI; 1989) utilizan definiciones diferentes. Conviene aquí señalar la importancia de revisar en el futuro próximo la forma de calcular el índice â en los reglamentos mexicanos.

De cualquier manera, no se puede considerar que ésta sea una discusión concluida. En un trabajo reciente de Rosowsky et al., (1994) donde se comenta un artículo previo de Heger (1993), se indica que aún existe confusión en las bases para la selección de los factores de resistencia empleados actualmente para estructuras de acero. Rosowsky et al., (1994) señalan con toda corrección que 
trabajos diferentes parecen sugerir niveles de confiabilidad diferentes utilizados para la calibración en el desarrollo de criterios probabilísticos para diseños de estructuras de acero (Ellingwood et al., 1980; Galambos, 1991; AISC, 1986). De cualquier modo parece que se empleó un índice de confiabilidad esperado de 2.6 para el caso del esrado límite de flexión, similar al de los reglamentos para madera en Norte América (Foschi, et al., 1989). Por su parte, Gromala (1993) emplea un valor de $\hat{a}=3.27$ para el caso de madera para andamios y también utiliza una ecuación del índice de confiabilidad, de la misma forma que los reglamentos mexicanos, aunque él utilizó la extensión para variabilidad alta de la formulación del índice de confiabilidad.

Ruiz y Aguilar (1994) ofrecen otras posibles explicaciones para la diferencia de valores de â entre los reglamentos mexicanos y los extranjeros, cuando menos para el caso de las estructuras de concreto reforzado: 1) Las probabilidades de excedencia de las cargas máximas son drásticamente diferentes entre el código mexicano (RCDF -87: DDF, 1987) y el de la ACI (1989). Ruiz y Aguilar recomiendan verificar si la probabilidad de excedencia planteada en el RCDF - 87 es adecuada; 2) $\mathrm{ACl} 318$ - 89 define la resistencia del material de manera diferente al RCDF - 87; 3) finalmente, los factores de carga y resistencia son diferentes entre ambos reglamentos, lo cual, argumenta Ruiz y Aguilar, también contribuye a la diferencia en valores de â. Como colofón de esta discusión sobre el índice de confiabilidad â para estructuras de madera, parece que lo más aconsejable es usar los métodos más avanzados y robustos posibles para el cálculo del índice de confiabilidad â, como por ejemplo, la formulación de Rackwitz y Fiessler (1978). De esta forma se pueden hacer comparaciones válidas entre los distintos materiales y se puede hacer una estimación adecuada de la probabilidad de falla $P_{f}$ de acuerdo con la Ec. (7).

\section{Factores de carga}

El lado derecho de la ecuación (10) está establecido en los reglamentos de cada país o cuidad y los factores de carga $F_{c}$ son comunes para todos los materiales. En el caso de la Ciudad de México, las recomendaciones generales del reglamento para el diseño de estructuras se incluye en el título VI "Seguridad Estructural de la Construcciones" del Reglamento para Construcciones del Distrito Federal (DDF, 1987 a). Las combinaciones de acciones que deben considerarse en el diseño se dividen en dos categorías, dependiendo de si intervienen en ellas acciones accidentales. De esta clasificación dependen los factores de carga que deben aplicarse para obtener las fuerzas internas de diseño.

El factor de carga para combinaciones que incluyan solamente acciones permanentes y variables es 1.4 , excepto cuando se trata de construcciones en que puede haber aglomeración de personas, en cuyo caso el factor de carga es de 1.5. Para combinaciones que incluyan alguna acción accidental, será 1.1 para todas las acciones. Para alguna acción cuyo efecto sea favorable para la seguridad de la estructura el factor de carga será 0.9. En la Tabla 1, usando la forma de presentación de Meli (1985) se presentan los factores de carga especificados por diferentes reglamentos para las combinaciones de carga más usuales que se presentan en el diseño.

\section{Factores de reducción de resistencia}

En el caso del Reglamento de Construcciones del DF, los factores de reducción se deben aplicarse a las resistencias, señalados en el lado 
Tabla 1. Factores de carga especificados por diferentes reglamentos

\begin{tabular}{|l|l|l|l|}
\hline REGLAMENTO & $\begin{array}{c}\text { COMBINACIONES } \\
\text { COMUNES }\end{array}$ & $\begin{array}{c}\text { COMBINACIONES } \\
\text { EXCEPCIONALES }\end{array}$ & $\begin{array}{c}\text { COMBINACIONES } \\
\text { PARA VOLTEO }\end{array}$ \\
\hline RDF 87 & $1.4 \mathrm{C}_{\mathrm{M}}+1.4 \mathrm{C}_{\mathrm{V}}(1)$ & $1.1 \mathrm{C}_{\mathrm{M}}+\mathrm{C}_{\mathrm{V}}+1.1 \mathrm{C}_{\mathrm{A}}(2)$ & $0.9 \mathrm{C}_{\mathrm{M}}+1.1 \mathrm{C}_{\mathrm{A}}$ \\
ACI 77 & $1.4 \mathrm{C}_{\mathrm{M}}+1.7 \mathrm{C}_{\mathrm{V}}$ & $1.5 \mathrm{C}_{\mathrm{M}}+1.275 \mathrm{C}_{\mathrm{V}}+1.275 \mathrm{C}_{\mathrm{A}}$ & $0.9 \mathrm{C}_{\mathrm{M}}+1.3 \mathrm{C}_{\mathrm{A}}$ \\
ASCE 7-88 & $1.2 \mathrm{C}_{\mathrm{M}}+1.6 \mathrm{C}_{\mathrm{V}}$ & $1.2 \mathrm{C}_{\mathrm{M}}+0.5 \mathrm{C}_{\mathrm{V}}+1.5 \mathrm{C}_{\mathrm{A}}(3)$ & $0.9 \mathrm{C}_{\mathrm{M}}+1.5 \mathrm{C}_{\mathrm{A}}$ \\
CEB 80 & $1.25 \mathrm{C}_{\mathrm{M}}+1.45 \mathrm{C}_{\mathrm{V}}$ & $1.25 \mathrm{C}_{\mathrm{M}}+\mathrm{C}_{\mathrm{V}}+\mathrm{C}_{\mathrm{A}}$ & $0.8 \mathrm{C}_{\mathrm{M}}+1.45 \mathrm{C}_{\mathrm{A}}$ \\
NRC 85 & $1.25 \mathrm{C}_{\mathrm{M}}+1.5 \mathrm{C}_{\mathrm{V}}$ & $1.25 \mathrm{C}_{\mathrm{M}}+1.05 \mathrm{C}_{\mathrm{V}}+1.05 \mathrm{C}_{\mathrm{A}}$ & $0.85 \mathrm{C}_{\mathrm{M}}+1.5 \mathrm{C}_{\mathrm{A}}$ \\
OHBDC 83 & $1.2 \mathrm{C}_{\mathrm{M}}+1.4 \mathrm{C}_{\mathrm{V}}(4)$ & $-1 .---(5)$ & $0.9 \mathrm{C}_{\mathrm{M}}+1.3 \mathrm{C}_{\mathrm{A}}(6)$ \\
\hline
\end{tabular}

Los factores de carga se aplican a las fuerzas internas debidas respectivamente a: acciones permanentes (carga muerta), $\mathrm{C}_{\mathrm{M}}$; acciones variables (carga viva), $\mathrm{C}_{\mathrm{V}}$; acciones accidentales (sismo o viento), $\mathrm{C}_{\mathrm{A}}$

\footnotetext{
1 Ambos factores de carga aumentan a 1.5 para edificaciones muy importantes

2 Cv es el valor instantáneo de la carga viva del diseño reducida

3 El factor 1.5 se aplica a CA si es debida al sismo; si es por viento se toma 1.3

4 Más cargas de empuje de tierras, presión hidrostática y efectos secundarios del preesfuerzo

5 Este reglamento para puentes no considera esta combinación de cargas

6 En este caso de suman otras cargas como las de presión del hielo, y las cargas por colisión
}

izquierdo de la ecuación (10), se consignan en las Normas Técnicas Complementarias para cada material y estado límite específicos. Los factores de reducción varían según el grado de seguridad y la precisión de la fórmula empleada para el cálculo, según el tipo de falla esperado, dúctil o frágil y, según el tipo de elemento estructural. En estas, los requisitos siguen un criterio común de estados límite en que las acciones y los factores de carga son iguales para todos los materiales (con algunas excepciones para el caso de estructuras ligeras de madera, para las cuales las cargas concentradas de diseño son menores) y, los factores de reducción de resistencia están determinados para lograr niveles de confiabilidad semejantes (Meli, 1985). En la Tabla 2 se incluyen los valores de los factores de reducción de resistencia de los reglamentos de diseño de Estados Unidos (ASCE, 1990) y Canadá (CSA, 1984) y el de la Ciudad de México (DDF, 1987b).

\section{Relación entre â y $\mathbf{F}_{\mathbf{R}}$}

Para ilustrar esta relación, se usará un ejemplo presentado por Foschi, et al., 1989. Primero se ajustaron las curvas de distribución a los datos utilizando el rango de datos completo. En la figura 4 se presenta el efecto de utilizar cuatro tipos de distribución estadística, sobre la relación entre $\beta$ y $F_{\mathrm{R}}$. Es aparente que la relación entre el índice de confiabilidad y el factor de resistencia es altamente 
Tabla 2. Factores de reducción para madera (DFCR)

\begin{tabular}{|l|c|c|c|}
\hline & ASCE & CSA & DDF \\
\hline Flexión & 0.85 & 0.70 & 0.80 \\
\hline Compresión & 0.90 & 0.75 & 0.70 \\
\hline Tensión & 0.80 & 0.70 & 0.70 \\
\hline Cortante & 0.75 & 0.60 & 0.70 \\
\hline Conectores & 0.65 & 0.70 & 0.70 \\
\hline
\end{tabular}

dependiente del tipo de distribución utilizado. En este caso, la distribución lognormal resulta menos conservadora, mientras que la distribución normal produce el más conservador para un factor de resistencia $F_{R}$ dado. Las distribuciones Weibull producen resultados intermedios.

Aunque pudiera argumentarse que los cuatro tipos de distribución podrían dar buenos ajustes del conjunto de datos, los cuatro difieren en su representación de la cola inferior, donde se ubican los valores de diseño. Para eliminar esta ambigüedad, es necesario enfocar la atención en dicha cola inferior, conservando, claro está, un número suficiente de puntos en el conjunto a analizar. Con los resultados presentados en la figura 5 se puede estudiar el caso de utilizar las cuatro distribuciones de probabilidad diferentes ajustadas únicamente a una fracción inferior de los datos de prueba ilustra la relación entre $F_{R}$ y â correspondiente al ajuste de los datos truncados al $15^{\circ}$ percentil. Se puede observar que no tiene mucho efecto sobre el índice de confiabilidad â, el utilizar cualquiera de las cuatro distribuciones estudiadas (normal, lognormal, Weibull de dos parámetros y
Weibull de tres parámetros) y que los resultados se vuelven más estables y menos influenciados por el tipo de distribución ajustado a la cola inferior (Foschi, et al., 1989).

\section{Efecto de la duración de carga sobre la resistencia}

Es importante señalar que la resistencia nominal indicada en la ecuación (10), $R_{n}$, se basa en un percentil inferior de la resistencia a corto plazo para la condición de uso requerida; para el caso de la madera, comúnmente se toma el valor del $5^{0}$ percentil. La resistencia de diseño debe incluir el efecto de duración de carga (DC). Esto se hace modificando la resistencia nominal por el factor de duración de la carga, $K_{D}$. Este factor es una modificación del factor de resistencia o comportamiento $F_{R}$. El procedimiento básico se ilustra en la figura 6 , la cual indica la relación entre el factor de resistencia $F_{R}$ y el índice de confiabilidad $a ̂$ para dos casos: uno, cuando los efectos de duración de carga se ignoran y únicamente se considera la resistencia a corto plazo y otro, cuando sí se consideran los efectos de la duración de carga sobre la resistencia. 


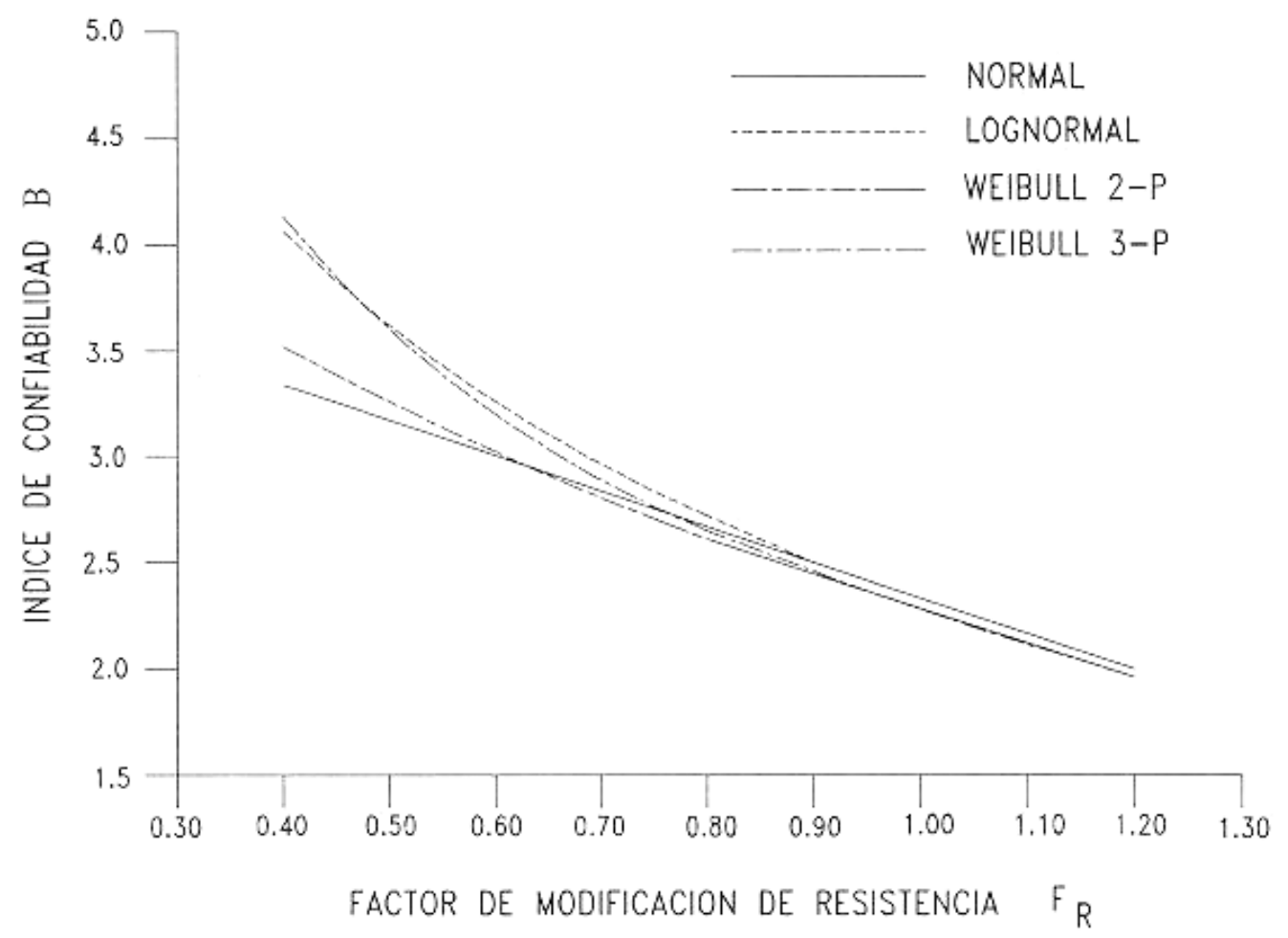

Figura 4. Relación entre $\beta$ y $F_{R}$ con cuatro tipos de distribución de probabilidad ajustadas al $100 \%$ de los datos (Foschi et al., 1989)

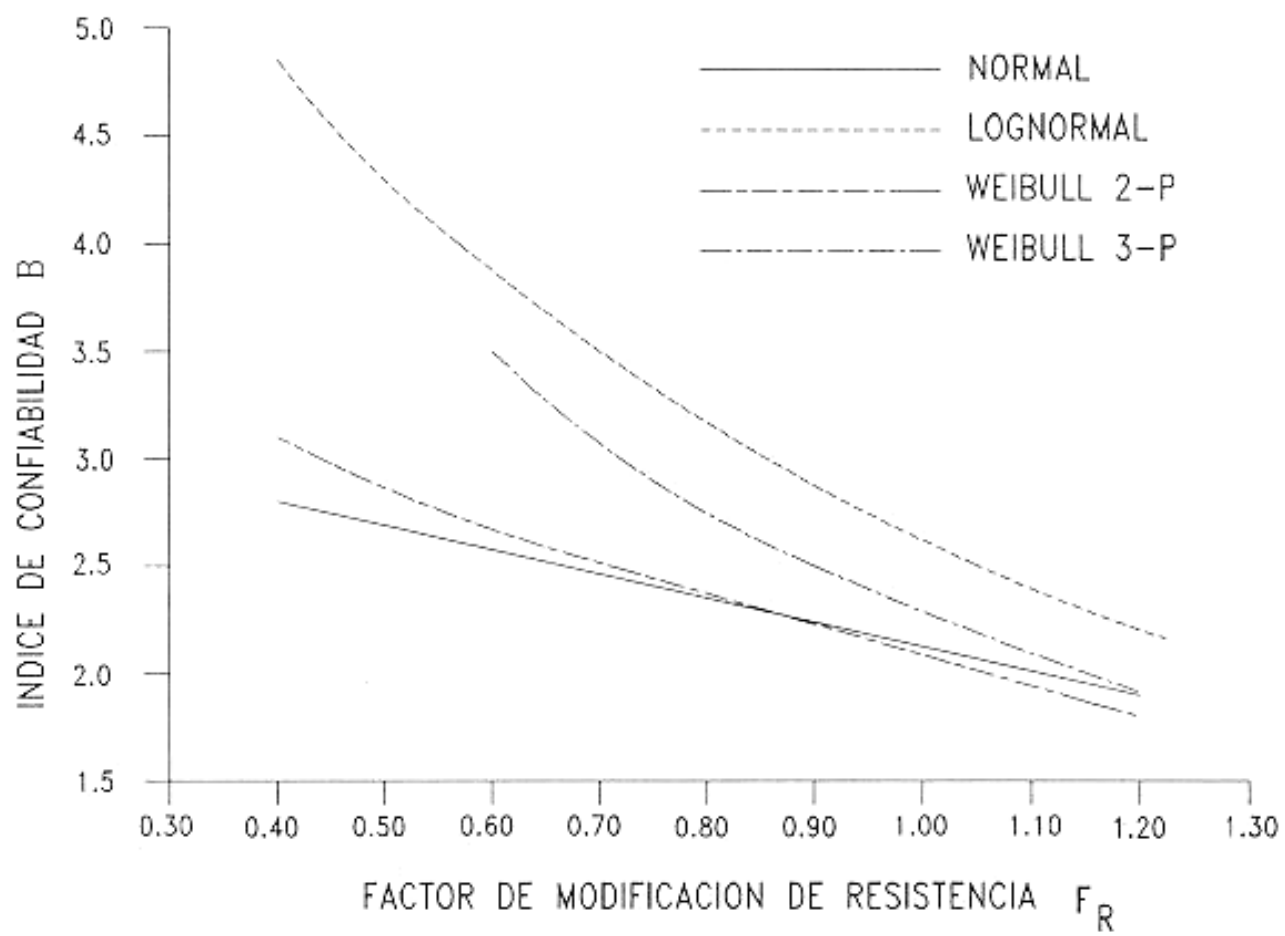

Figura 5. Relación entre $\beta$ y $F_{R}$ con cuatro tipos de distribución de probabilidad ajustadas al 15\% de los datos (Foschi et al., 1989) 


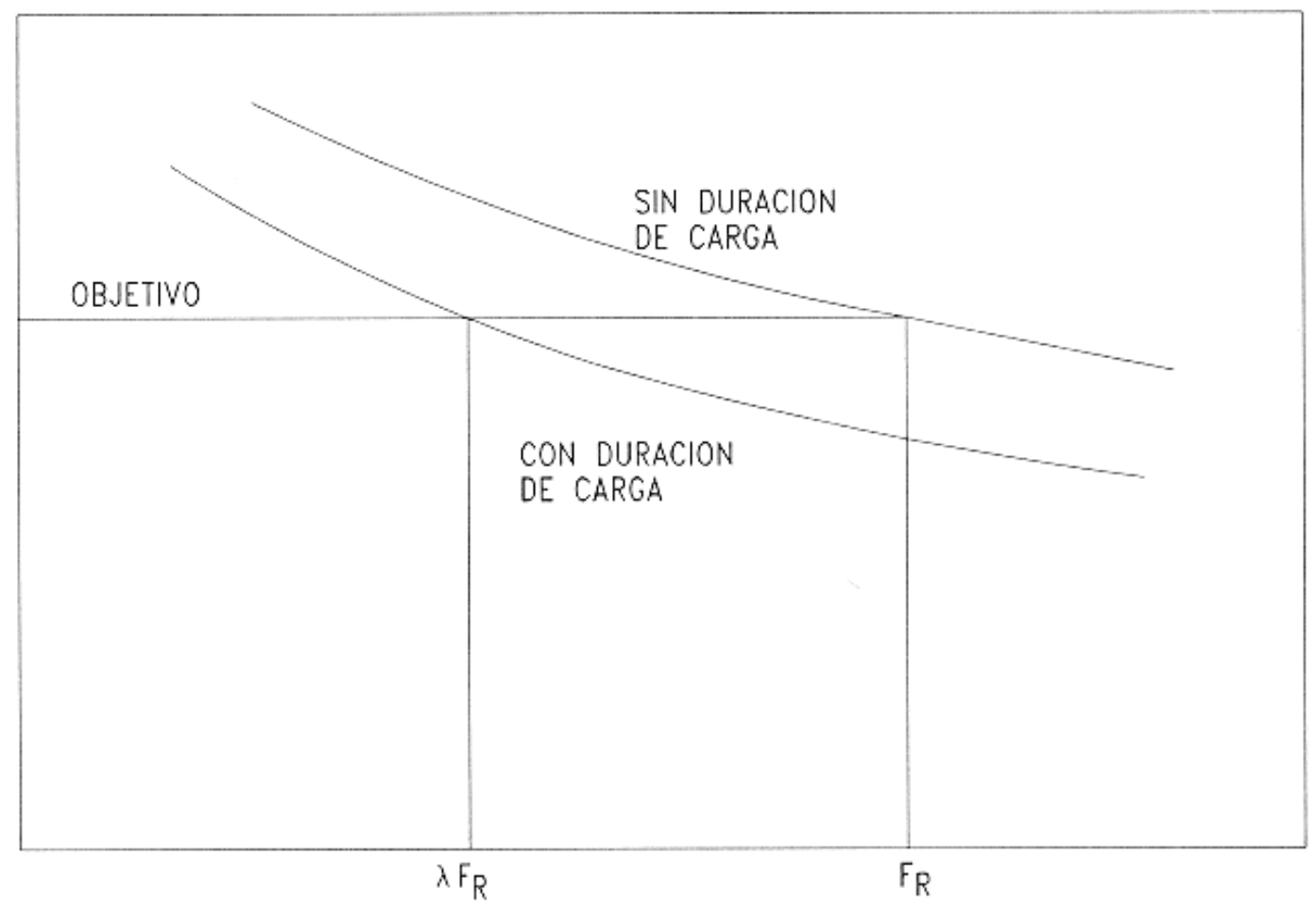

Figura 6. Determinación del factor del efecto de carga para mantener una misma Confiabilidad (Ellingwood, 1992)

En el caso de las NTCM (DDF, 1987 b), para el cálculo de los factores de reducción de resistencia se consideró la resistencia de la madera a corto plazo, la cual se comparó con las cargas máximas que se espera ocurran durante la vida útil de la estructura. El efecto de la duración de carga sobre la resistencia se tomó en cuenta reduciendo el valor de esfuerzo para cargas de corta duración por el factor de duración de carga tradicional para la condición de carga normal (1/1.6) de manera que los valores especificados en las tablas ya reflejan esta reducción (Robles, et al., 1988).

En la tabla 3 se presentan diferentes valores de los factores de duración de carga, $\mathrm{K}_{\mathrm{D}}$, para las diferentes condiciones de carga. Los datos de las dos primeras columnas son de resultados experimentales obtenidos en dos laboratorios (USFPL, Madison, Wi. y University of Bristish Columbia (UBC), Vancouver, Canadá) presentados por Ellingwood y Rosowsky (1991), los de la columna 3 son valores propuestos por Ellingwood (1992) y los de la columna 4, son los valores adoptados por el DDF (1987b).

\section{COMENTARIOS ACERCA DEL DISEÑO PROBABILISTICO}

Un argumento a favor del diseño probabilístico es que provee una medida cuantitativa de la seguridad. Esto ha generado un interés natural en tratar de validar los métodos basados en la confiabilidad a través de la comparación 
Tabla 3. Factores de efecto del tiempo, $K_{p}$, DFCR

\begin{tabular}{|l|c|c|c|c|}
\hline COMBINACION CARGA & USFPL & UBC & ELLINGWOOD & DDF \\
\hline $\mathrm{C}_{\mathrm{M}}$ (únicamente) & 0.59 & 0.50 & 0.60 & 0.56 \\
\hline $\mathrm{C}_{\mathrm{M}}+\mathrm{C}_{\mathrm{V}}$ (ligera) & 0.76 & 0.80 & 0.80 & 0.62 \\
\hline $\mathrm{C}_{\mathrm{M}}+\mathrm{C}_{\mathrm{M}}$ (pesada) & 0.67 & 0.80 & 0.70 & 0.62 \\
\hline $\mathrm{C}_{\mathrm{M}}+\mathrm{C}_{\mathrm{V}}$ (techos) & 0.78 & 0.80 & 0.80 & 0.77 \\
\hline $\mathrm{C}_{\mathrm{M}}+$ Viento & $>1.0$ & 1.00 & 1.00 & 0.82 \\
\hline Impacto & $>1.0$ & 1.00 & 1.00 & 0.99 \\
\hline
\end{tabular}

$\mathrm{C}_{\mathrm{M}=\text { carga muerta } \quad \mathrm{C}_{\mathrm{V}}=\text { carga viva }}$

con tasas observadas de falla. Sin embargo, las diferencias entre las tasas observadas de falla y las predichas a menudo son substanciales (Ellingwood, 1992). En lo que sigue se comentan algunas de las razones que podrían explicar estas discrepancias.

Los estudios de seguridad estructural inevitablemente están relacionados con eventos raros, por lo que es necesario hacer inferencias con muestras pequeñas. Esto ha dado como resultado que los intervalos de confianza para estimadores de confiabilidad sean relativamente grandes. Por otro lado, las combinaciones de carga empleadas en el diseño reflejan únicamente los efectos de variabilidad estocástica en las cargas. Sin embargo, la mayoría de las fallas en las estructuras (80 a $90 \%$ ) se deben a errores al diseño, planeación y construcción, como se ilustra en la figura 7 , tomada de un trabajo de Matousek y Schneider (1976). Esto significa que la gran mayoría de las causas de falla ocurre en áreas bastante diferentes a aquellas en que los métodos probabílisticos son útiles. Los factores de seguridad juiciosamente seleccionados cubren solamente errores menores y no las debidos a errores humanos que son las causa de las fallas más notables.

Sin embargo, Gromala y Murphy (1988) al discutir este mismo tema, presentan datos de Thoft - Christensen y Baker (1982) quienes atribuyen a falta de experiencia el $60 \%$ de fallas estructurales por ellos estudiadas (120 casos). Gromala y Murphy comentan que el DFCR representan un gran avance en cuanto a proveer calidad en las construcciones de madera por varias razones, entre ellas el de ser más fácil de aprender y enseñar, lo cual contribuiría a que hubiera menos fallas de las construcciones.

Los métodos de confiabilidad actualmente en uso se han efectuado a tratamiento de elementos aislados más que al comportamiento de sistemas estructurales. Aunque esto no es más que un reflejo de la práctica tradicional, es de esperarse que el análisis de confiabilidad de sistemas arroje diferencias importantes con respecto al análisis de elementos individuales. Para lograr una mayor congruencia entre los métodos de análisis 
Madera y Bosques 2(1), 1996:9-31

$y$ el comportamiento real de las estructuras, en el análisis de sistemas estructurales reviste capital importancia el identificar los modos o trayectorias de falla para poder formular los estados límite apropiados a un sistema tomado como conjunto (Ellingwood, 1992).

\section{CAUSAS DE DAÑOS EN LAS CONSTRUCCIONES}

\section{CAUSAS DE DAÑOS EN LAS CONSTRUCCIONES}

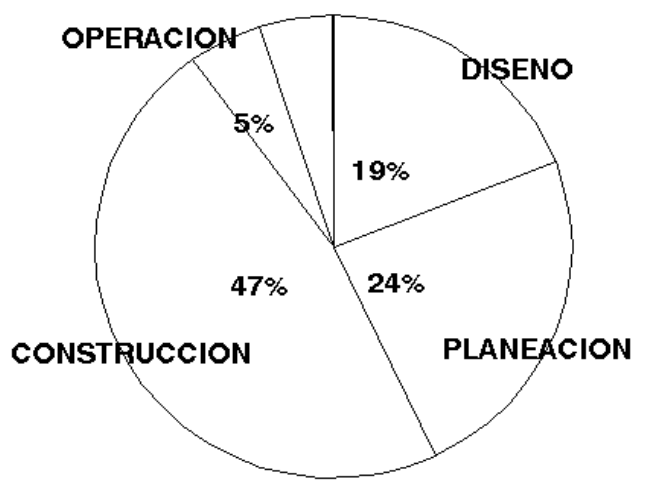

Figura 7. Causas de daños en las estructuras (Matousek y Schneider, 1976)

\section{ESTADOS LIMITE DE SERVICIO}

Después del objetivo básico de obtener una seguridad adecuada, el segundo objetivo del diseño estructural es lograr un comportamiento satisfactorio en condiciones normales de operación de la construcción, lo mismo que ante acciones accidentales que pudieran presentarse durante la vida útil de la misma. Esto equivale a que debe haber una probabilidad pequeña de que la respuesta rebase los estados límite de servicio. En el proceso de diseño debe revisarse que no excedan estos, para lo cual se emplearán las acciones sin ser afectadas por factores de carga (Meli, 1991).
Los estados límite de servicio se vuelven cada vez más importantes en las estructuras modernas flexibles (ASCE, 1986). Los requerimientos actuales de limitar las deflexiones estáticas debidas a las carga viva a una fracción del claro (1/240 ó1/360, por ejemplo), se han usado por más de 150 años (Ellingwood, 1992) y, por muchas aplicaciones, podrían ser insuficientes para garantizar un comportamiento funcional de las estructuras, particularmente porque muchos de los problemas de servicio con las estructuras de madera se refieren a vibraciones indeseables (Ohlsson, 1982; Foschi, et al., 1993).

\section{REGLAMENTOS DE ESTADOS LIMITE PARA MADERA}

Con un mejor entendimiento del efecto de duración de carga, la disponibilidad de modelos de carga estocásticos, y los resultados de los programas de evaluación de la resistencia de la madera en tamaño estructural (Green y Evans, 1987; Echenique-Manrique et al., 1987 , Cerón, 1988), la preparación de reglamentos para productos de madera de usos estructurales ha avanzado en la misma dirección que el acero y el concreto.

En los Estados Unidos un comité de especialistas se llevó dos años para completar un borrador de reglamento de construcciones para estructuras de madera basado en criterios probabilísticos (Murphy et al., 1988). Esta versión toma en cuenta de una manera más realista y consistente la variabilidad inherente en la resistencia y rigidez de los productos de madera, así como la naturaleza dependiente del tiempo de la resistencia de los mismos bajo condiciones de carga estocástica. Otra ventaja de este reglamento es que utiliza enfoques modernos para las ecuaciones de columnas, vigas y conexiones (Zahn, 
26

1988; McLain y Carroll, 1990). Este borrador fue revisado posteriormente y se tenia la esperanza en el año 1993 se adoptara un reglamento de consenso (es decir nacional) (ASCE,1993), lo cual si bien no sucedió está muy próximo a convertirse en realidad.

En contraparte, el grupo de investigadores mexicanos encargados de la elaboración de un reglamento similar se llevo los mismos dos años en concluir un reglamento final, iniciando de cero, puesto que la versión anterior estaba basado en conceptos de esfuerzos permisibles, (Robles et al.;1988). Las formulas de cálculo adoptadas en el reglamento mexicano son muy similares a las del reglamento para diseño de puentes carreteros de Ontario, Canadá (OMTC, 1983). En México, desde 1987 está en vigor un reglamento de construcciones para madera basado en la confiabilidad (DDF, 1987b).

En Canadá, un reglamento de construcciones de éstas características tiene varios años en uso (CSA, 1984), después de haber acumulado una gran cantidad de información sobre aspectos fundamentales del comportamiento estructural de la madera (De Grace, 1986). Esta dirigido principalmente al diseño de edificaciones y sus requerimientos de carga son idénticos a los del reglamento nacional canadiense (NRC, 1985), aunque también puede utilizarse para el diseño de puentes, torres, tanques, muros de contención, y estructuras de todo tipo, siempre que se cuente con la información necesaria sobre las cargas.

Los reglamentos, basados en el criterio de estados límite tienen la ventaja sobre anteriores reglamentos, de que cuentan con bases más sólidas para las ecuaciones de diseño. Por otro lado, las fuentes de
Diseño probabilístico. Dávalos

incertidumbre que se deben considerar son mas aparentes al ser tratadas en forma más explícita que en los reglamentos basados en el método de esfuerzos permisibles. Finalmente, en lo que constituye una de las ventajas más interesantes de los enfoques más recientes, los métodos probabilísticos resultan mas fáciles de enseñar lo que en última instancia facilitará la introducción del diseño con madera a una mayor escala (Ellingwood, 1992).

\section{CONCLUSIONES}

Las conclusiones a las que se llegaron en el Taller TIA-OTAN pueden hacerse extensivas a éste documento sobre estado del conocimiento de los métodos de diseño probabilístico en América del Norte, citadas a continuación:

$\risingdotseq$ Es necesario buscar la armonización de los reglamentos de construcción de los diferentes países. En Europa han dado un gran paso a éste respecto con el reglamento supranacional Eurocode (CEC, 1987).

$\equiv$ Debe tenderse a la formación de bases de datos internacionales, que puedan ser intercambiadas entre investigadores de diferentes países.

$\equiv$ Finalmente, pero no por ello menos importante debe buscarse el mejoramiento de las comunicaciones internacionales entre los investigadores.

\section{RECONOCIMIENTOS}

Este trabajo se realizo con fondos fiscales del Departamento de Productos Forestales y Conservación de Bosques del Instituto de Ecología, con la clave 902-13. 
Madera y Bosques 2(1), 1996:9-31

\section{REFERENCIAS}

American Concrete Institute 1989. Building code requirements for reinforced concrete, $\mathrm{ACl}$ 318-89, American Concrete Institute $(\mathrm{ACl})$, Detroit, Mich.

American Society of Civil Engineers. 1990. Minimum design loads for buildings and other structures (ASCE 7-88). Nueva York. (Anteriormente Am. Nat. Std. A58.1-1982).

American Society of Civil Engineers. 1993. Progress reported on LRFD Wood Standard by ASCE Codes Group. ASCE News, Abril 18(4):8.

Barrett, J.D. y R.O. Foschi. 1979. Duration of load and probability of failure in wood. Parts I and II. Canadian Journal of Civil Engineering, 5(4): 505-532.

Bodig, J., Ed. 1992. Reliability-based design of engineered wood structures. NATO ASI Series E: Applied Sciences, Vol.215. Kluwer Academic Publishers, Dordrecht, Holanda.

Boyd, C.W. P. Koch, H.B. McKean, C.R. Morschauser, S.B. Preston y F.F: Wangaard. 1976. Wood for structural and architectural purposes. Wood and Fiber 8(1):3-72.

Canadian Standards Association. 1984. CAN3-086. 1-M84. Engineering design in wood (Limit states design). Canadian Standards Association, Rexdale, Ont., Canadá.

Cerón C., M. 1988. Calificación, Clasificación y obtención de valores de diseño de maderas tropicales Mexicanas. Informe técnico final.
Facultad de Ingeniería, Universidad Autónoma de Yucatán. Mérida, Yuc.

Chávez G.A 1985. Análisis de las propiedades mecánicas en flexión estática de tableros contrachapados de madera de pino. Tesis profesional, EITECMA, Universidad Michoacana de San Nicolás de Hidalgo, Morelia, Mich.

Commission of the European Communities. 1987 Eurocode 5, Design of Timber Structures, Report EUR 9887.

Dávalos S, R., F.F. Wangaard y R Echenique M. 1977. Clasificación de La madera de pinos mexicanos. La Madera y su Uso en la Construcción No.2. INIREB-LACITEMA. Xalapa, Ver.

Dávalos S.R. 1994. Ayudas de diseño. In: M. Elorza, de. Manual de Construcción de Estructuras Ligeras de Madera (COFAN), México, D.F.

De Grace, R.F. 1986. Commentary on CSA Standard CAN3-086.1-M84 Engineering design in wood (Limit states design). Canadian Standards Assn, Rexdale, Ontario, Canadá.

Departamento del Distrito Federal. 1976. Reglamento de Construcciones para el Distrito Federal. Publicado en el Diario Oficial de la Federación, 15 de diciembre de 1976, México, D.F.

Departamento del Distrito Federal. 1987a.Reglamento de Construcciones para el Distrito Federal. Editorial Porrúa, S.A. Décima Edición (1989), México,D.F.

Departamento del Distrito Federal. 1987b. Normas técnicas complementaras para el diseño y construcción de estructuras de madera. Gaceta Oficial 
del Departamento del Distrito Federal, Quinta época No. 48, 10 de diciembre de 1987, México, D.F.

Echenique M., R, R Dávalos S. y F. Robles F.-V 1987 Informe final al CIID. Calificación y Clasificación de madera de pino para usos estructurales (Timbergrading-México). Fase III INIREB-LACUTEMA, Xalapa, Ver.

Ellingwood, B.R., Galambos, J.G. Mcgregor y C.A. Coronell. 1980. Development of a probability based load criterion for American National Standard A58, Building code requirements for minimum design loads in buildings and other structures. NBS Special Publication 577, U.S. Department of Commerce, National Bureau of Standards, Washington, D.C.

Ellingwood, B.R., y D Rosowsky, 1991. Duration of load effects in LRFD for wood construction. Journal of Structural Engineering, ASCE 117(2):584-599.

Ellingwood, B.R. 1992. Status of reliabilitybased design in North America: Impact for engineered wood construction. In: J. Boding, de. Reliability-based design of engineered wood structures. NATO ASI Series E: Applied Sciences, Vol. 215 Kluwer Academic Publishers, Dordrecht Holanda.

Foschi, R.O. 1979. A discussion on the applications of the safety index concept to wood structures. Canadian Journal of Civil Engineering 6(1):51:58.
Diseño probabilístico. Dávalos

Foschi, R.O. 1992 Material characteristics and reliability-based design. In: J. Boding, de reliability-based design of engineered wood structures. NATO ASI Series E: Applied Sciences, Vol. 215. Kluwer Academic Publishers, Dordrecht, Holanda.

Foschi, R.O., B.R. Folz y F.Z. Yoa. 1989. Reliability-based design of wood structues. Structural Research Series, Report No. 34, Dept. Of Civil Eng., University of British Columbia, Vancouver, B.C., Canadá

Foschi, R.O., G.A. Numan, B.R. Folz y F.Z. Yoa. 1993. Floor vibration due to occupants: experimental, theoretical and reliability-based results. Structural Research Series Report, Dept. Of Civil Eng., University of British Columbia, Vancouver, B.C., Canadá

Freudenthal, A., J. Garrelts y M. Shinozouka. 1966. The analysis of structural safety. Journal of Structural Engineering, ASCE 92(1):267-325.

Galambos, T.V. 1991. Lessons from five years of LRFD in steel structures. Proc., William McGuire Symposium, Cornell University, Ithaca, N.Y.

Gerhards, C-y C- Link. 1986. Effect of loading rate on bending strength of Douglas-fir 2 by 4's". Forest Products Journal, 36(2):63-66

Green, D.W. y J.W. Evans. 1987. Mechanical properties of visually grades lumber, Vols. 1-5. USDA Forest Service, Forest Products Laboratory, Madison, W..I.

Gromala, D.S. 1993. Practical application of reliability-based design concepts to wood scaffold planks. Forest Products Journal 43(9):29-34. 
Gromala, D.S. y J.F. Murphy. 1988. Benefits of LRFD and opportunities. In: J.F. Murphy, De. Load and resistance factor design for engineered wood construction - a prestandard report. American Society of Civil Engineers, Nueva York, NY.

Heger, F.J. 1993 Public safety - is it compromised by new LRFD design standards Journal of Structural Engineering 119(4): 1251 - 1264.

Madsen, B. y J.D. Barrett. 1976. Duration of load. Forest Products Journal 26(2):57

Madsen, H.O., S. Krenk y N.C. Lind. 1986. Methods of structural safety. PrenticeHall, Inc., Englewood Cliffs, N.J.

Matousek, M. y J. Schneider. 1976. Untersuchungen zur Struktur des Sicherhitsprolblems bei bauwerken (Investigaciones acerca de la naturaleza de los problemas de la seguridad de las construcciones). Bericht No. 59 des Instituts für Baustatik und Konstuktion der ETH Zürich. Birkhäuser Verlang, Basel und Stuttgart.

McClain, T.E. y Carroll. 1990. Combined Load capacity of threaded fastener wood connections. Journal of Structural Engineering, ASCE 116(9): 2419-2432

Meli P., R. 1976. Bases para los criterios de diseño estructural del reglamento de construcciones para el Distrito Federal. Ingeniería 46(2):137-157

Meli P., R. 1985 Diseño estructural Limusa, México, D.F.
Meli P.R. 1991 comentarios al título sexto "Seguridad estructural de las construcciones" del reglamento de construcciones para el Distrito Federal. Series del Instituto de Ingeniería UNAM No. ES - 1, México, D.F.

Murphy, J.F., de.. 1988. Load and resistance factor design for engineered wood construction - a prestandard report. American Society of Civil Engineers. Nueva York, NY.

National Research Council of Canada. 1985. National Building Code of Canada. NRC, Ottawa, Canadá.

Nielsen, L.F. 1984. Power law creep as related to relaxation, elasticity, damping rheological spectra and creep recovery - with special reference to wood. Memorias de la reunión de Grupo de Ingeniería de la Madera de IUFRO, Xalapa, Ver. Pp: 181-204

Ohlsson, S. 1982. Floor vibrations and human discomfort. Chalmers University of Technology, Dept. of Structural Engineering, Div. of Steel and Timber Structures, Gotemburgo, Suecia.

Ontario Ministry of Transportation and Communications. 1983. Ontario highway bridge design code. Highway Engineering Division, OMTC, Toronto, Ontario, Canadá.

Rackwitz, R. y B. Fiessler. 1978. Structural reliability under combined random load sequence. Computers and Structures 9:489-494. 
30

Robles F.-V., F., R. Dávalos S. y M.O. Ricalde C. 1988. Comentarios a las normas técnicas complementarias ara diseño y construcción de estructuras de madera, versión 1987. La Madera y su Uso No. 15 INIREB-LACITEMA, Xalapa, Ver.

Rosenblueth, E. y L: Esteva. 1973. Reliability basis for some Mexican codes. In: Probabilistic design of reinforced concrete buildings, $\mathrm{ACI}, \mathrm{SP}$ 31, Detroit, MI.

Rosowsky, D.V., A.F. Hassan y N.V.V. Phani Kumar. 1994. Calibration of current factors in LRFD for steel. Journal of Structural Engineering 120(9):2737-2746.

Ruiz, S.E. y J.C. Aguilar. 1994. Reliability of short and slender reinforced-concrete columns. Journal of Structural Engineering 120(6): 1850-1865.

Shinozouka, M. 1983. Basic analysis of structural safety. Journal of Structural Engineering, ASCE 109(3):721-740.

Thoft-Christensen, P. y M.J. Baker. 1982. Structural reliability theory and its applications. Springer-Verlag, N. Y.

U.S. Department of Commerce. 1991. United States industrial outlook. U.S. Government Printing Office, Washington, D.C.

Wood, L.W. 1951. Relation of strength of wood to duration of load. Reporte R1916, Forest Products Laboratory, Madison, Wi.

Zahn, J. 1988. Combined load stability criterion for wood beam columns. Journal of Structural Engineering, ASCE 114(11): 2612-2628.
Diseño probabilístico. Dávalos

\section{NOTACION}

$\mathrm{C}_{\mathrm{M}}=$ acciones permanentes (carga muerta)

$\mathrm{C}_{\mathrm{R}}=$ coeficiente de variación de la resistencia

$\mathrm{C}_{\mathrm{Q}}=$ coeficiente de variación del efecto de las cargas actuantes

$\mathrm{C}_{\mathrm{V}}=$ acciones variables (carga viva)

$\mathrm{C}_{\mathrm{A}}=$ acciones accidentales (sismo o viento)

$\mathrm{D}=$ Dominio de la región de $\mathbf{x}$ donde $\mathrm{G}(\mathbf{X})<0$

$\mathrm{F}_{\mathrm{C}}=$ factor de carga

$\mathrm{f}_{\mathrm{k}}=$ Esfuerzo debido a las cargas aplicadas

$\mathrm{F}_{\mathrm{k}}=$ esfuerzo resistente

$\mathrm{R}=$ factor de resistencia 0 comportamiento

$\mathrm{FS}=$ factor de seguridad

$f_{x}(\mathbf{x})=$ función de densidad conjunta de $\mathbf{X}$

$\mathrm{G}(\mathbf{X})<0$ = función que relaciona las variables de carga y resistencia para el estado límite específico de interés

$\mathrm{K}_{\mathrm{D}}=$ factor de efecto del tiempo sobre la resistencia. También llamado factor de duración de la carga.

$I$ = claro de diseño

$\mathrm{m}_{\mathrm{G}}=$ media de la función estado límite, $\mathbf{G}(\mathbf{X})$

$\mathrm{m}_{\mathrm{R}}=$ valor medio de la resistencia

$\mathrm{m}_{\mathrm{Q}}=$ valor medio del efecto de las cargas actuantes

$\mathrm{P}_{\mathrm{f}}=$ probabilidad de falla

$\mathrm{Q}_{\mathrm{ni}}=$ carga nominal

$r$ = razón de carga muerta a carga total (muerta más viva)

$\mathrm{R}=$ resistencia generalizada

$R_{n}=$ resistencia nominal

$\mathbf{X}=\left(X_{1}, X_{2}, \ldots, X_{m}\right)$ vector que representa las variables de carga y resistencia

â = índice de confiabilidad 
Madera y Bosques 2(1), 1996:9-31

$\hat{a}_{0}=$ índice de confiabilidad buscado

$\mathbf{v}=$ variable que relaciona la resistencia, $\mathrm{R}$, y las cargas, $Q$, y mide la seguridad de la estructura

Ö(· ) = función de probabilidad normal estándar acumulada Ö $^{-1}=$ inversa de la función de probabilidad normal estándar acumulada

ó O $_{\mathrm{G}}=$ desviación estándar de la función estado límite, $\mathrm{G}(\mathbf{X})$ 